Recebido em 04/2016. Aceito para publicação em 06/2016.

\title{
ISOLAMENTO VERTICAL DO MULTI-NESTED PENDULA PARA DETECTORES INTERFEROMÉTRICOS DE ONDAS GRAVITACIONAIS
}

\author{
VERTICAL ISOLATION OF MULTI-NESTED PENDULA FOR INTERFEROMETRIC \\ GRAVITATIONAL-WAVE DETECTORS \\ Elvis Camilo Ferreira ${ }^{1}$ \\ Márcio Constâncio Junior ${ }^{2}$ \\ Odylio Denys Aguiar ${ }^{3}$ \\ Allan Douglas dos Santos Silva ${ }^{4}$
}

Resumo: Neste trabalho apresentamos os primeiros resultados do estudo do isolamento vertical em um sistema de pêndulos multi-aninhados, para aplicação como isoladores vibracionais passivos dos espelhos da próxima geração de detectores interferométricos de ondas gravitacionais, como o LIGO Voyager. As funções de transferência teórica dos modos verticais indicam um fator de isolamento de até seis ordens de grandeza em $10 \mathrm{~Hz}$. As medições dessas oscilações apresentam frequências de ressonância indesejáveis e indicam que o sistema necessita de melhorias. Aqui propomos algumas soluções para isso, que já estão sendo estudadas.

Palavras-chave: ondas gravitacionais; detectores; LIGO; isolamento vibracional.

Abstract: In this work, we present the first results of a study of a vertical isolation of the multi-nested pendula, to be applied as passive isolation on the mirrors of the next generation of interferometric gravitational-wave detectors, such as LIGO Voyager. The vertical transfer functions indicate an attenuation factor of up to six orders of magnitude at $10 \mathrm{~Hz}$. The measurement of these oscillations shows undesirable resonance frequencies and indicates that the system needs improvement. Then, we propose some solutions to this problem, which are already being studied.

Keywords: gravitational waves; detectors; LIGO; vibration isolation.

\footnotetext{
${ }^{1}$ Doutorando em Astrofísica - Instituto Nacional de Pesquisas Espaciais - INPE, Brasil. E-mail: elvis.ferreira@inpe.br.

${ }^{2}$ Doutorando em Astrofísica - Instituto Nacional de Pesquisas Espaciais - INPE, Brasil. E-mail: marcio.constancio@inpe.br.

${ }^{3}$ Doutor em Física. Pesquisador titular do Instituto Nacional de Pesquisas Espaciais - INPE, Brasil. E-mail: odylio.aguiar@inpe.br.

${ }^{4}$ Graduando em Engenharia Mecânica - Instituto Nacional de Pesquisas Espaciais - INPE, Brasil. E-mail: allan_2901gl@hotmail.com.
} 


\section{INTRODUÇÃO}

As ondas gravitacionais são deformações no espaço-tempo que se propagam à velocidade da luz, segundo a Teoria da Relatividade Geral. Sistemas astrofísicos, como binárias compactas, além de supernovas, estrelas de nêutrons e buracos negros, são exemplos de sistemas geradores destas ondas (SATHYAPRAKASH; SCHUTZ, 2009). Logo, a astronomia de ondas gravitacionais pode revelar informações inéditas sobre o Universo, principalmente aquelas que não podem ser obtidas através de ondas eletromagnéticas.

Em fevereiro de 2016, a colaboração científica do LIGO (Laser Interferometer Gravitational-Wave Observatory) anunciou a detecção de um sinal de onda gravitacional proveniente da fusão de dois buracos negros (ABBOTT, 2016), inaugurando essa nova janela de observação.

Agora, é necessária a construção de observatórios cada vez mais sensíveis. Um dos principais desafios é a redução de ruídos vibracionais nos espelhos dos interferômetros, provenientes do solo, sísmicos, naturais, ou devido a atividades humanas ou animais.

A fim de minimizar esses ruídos, o Advanced LIGO, atual versão (segunda geração) do LIGO, possui sistemas ativos e passivos de isoladores vibracionais, formados por sensores, atuadores, sistemas massa-mola e sistemas pendulares (AASI, 2015).

Na curva de sensibilidade projetada para o Advanced LIGO (Figura 1) pode se que os ruídos sísmicos dominam e formam uma "barreira" abaixo de $10 \mathrm{~Hz}$.

Figura 1 - Curva de sensibilidade projetada para o Advanced LIGO.

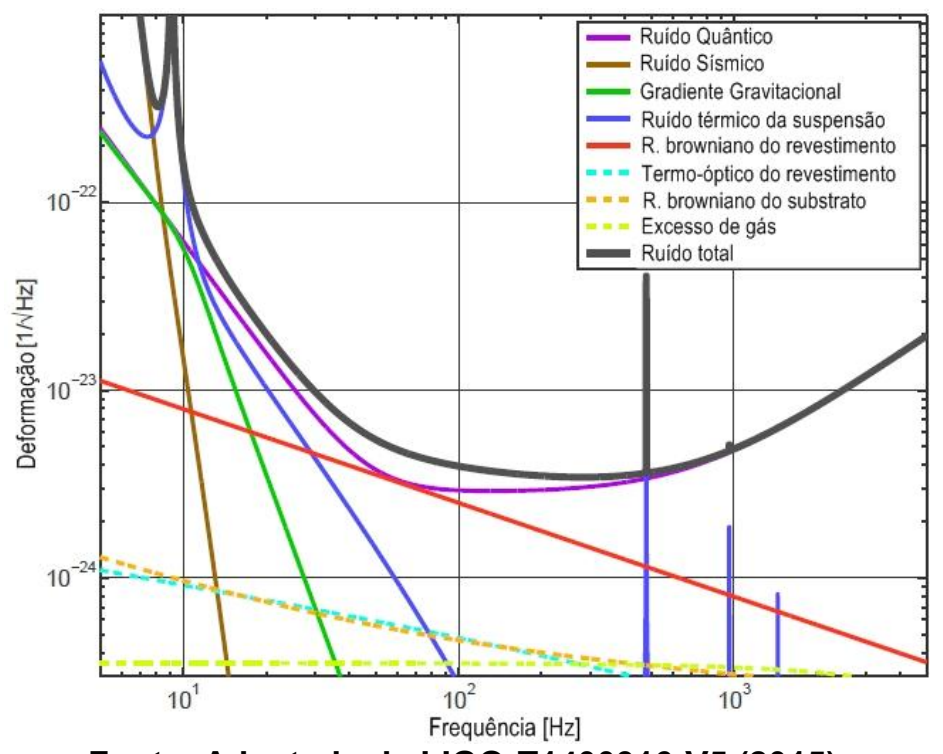

Fonte: Adaptada de LIGO-T1400316-V5 (2015). 
Com objetivo de filtrar ruídos vibracionais e tornar a terceira geração desse detector (LIGO Voyager) mais sensível em baixas frequências ( $<10 \mathrm{~Hz}$ ), o grupo de ondas gravitacionais da divisão de astrofísica do INPE (GWINPE) desenvolve, junto a colaboração LIGO, um sistema de pêndulos multi-aninhados com isolamento vertical, executado por molas.

Este artigo apresenta um modelo e os resultados dos primeiros testes de um sistema de pêndulos multi-aninhados com molas para isolamento vertical.

\section{DESENVOLVIMENTO}

\subsection{Multi-Nested Pendula}

O Multi-Nested Pendula (MNP) (CONSTANCIO JÚNIOR, 2013) consiste em um sistema pendular de cinco estágios, equivalente a um sistema em cascata, porém cada massa, que corresponde a uma casca cilíndrica de alumínio, está aninhada na massa do estágio anterior (Figura 2). Isto é, a massa de cada estágio é suspensa por intermédio de três hastes que conectam a parte superior de cada casca cilíndrica à parte inferior da casca imediatamente interna, e, assim, sucessivamente. As hastes são conectadas através de flanges superiores e inferiores, encaixados nos cilindros.

\section{Figura 2 - Primeiro protótipo do Multi-Nested Pendula.}

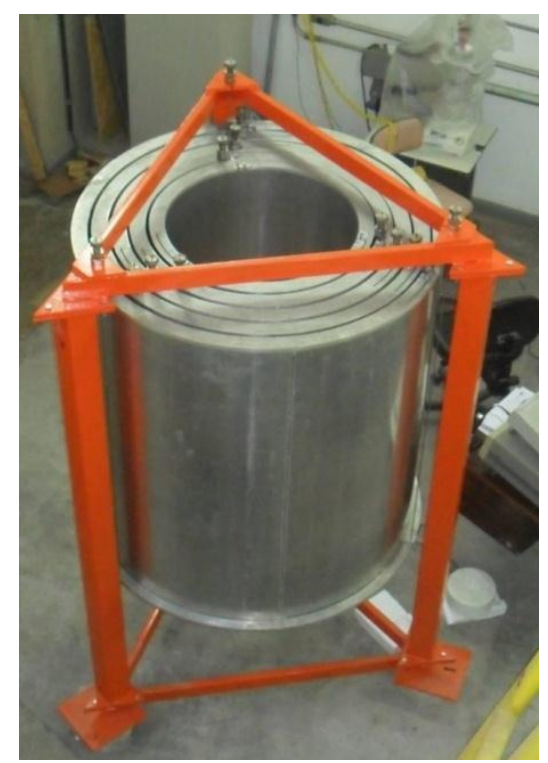

Fonte: Adaptada de Constancio Júnior (2013).

O fator de isolamento de um sistema multipendular é de, aproximadamente, $\left(f_{0} / f\right)^{2 N}$, para $f$ muito maior que a frequência de ressonância $\mathrm{f}_{0}$ dos estágios. Contudo, o ruído de vibrações verticais pode se converter em 
oscilações horizontais por um fator maior que $10^{-3}$ (SAULSON, 1994), levando a uma péssima eficiência do sistema. A fim de criar um isolamento vertical para o MNP, foram desenvolvidas e adicionadas molas de ar (FERREIRA, 2014), conforme a Figura 3.

Figura 3 - Visão de corte do Multi-Nested Pendula com as molas de ar instaladas, feita no Solid Works.

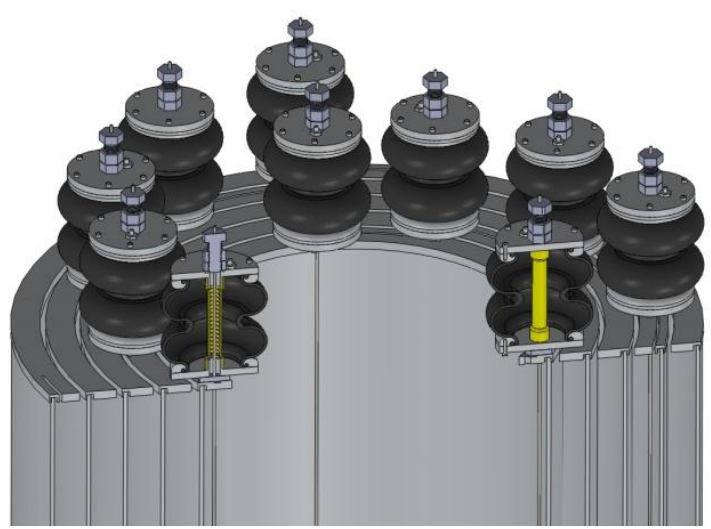

Fonte: Adaptada de Ferreira (2014).

\subsection{Molas de ar}

As molas de ar, desenvolvidas pelo GWINPE, são formadas de um fole de borracha, anéis para o encaixe das tampas e uma mangueira e mola internas para passagem da haste do MNP. Elas funcionam por compressão, de forma que o peso é aplicado na superfície (tampa) superior. Ao todo, foram construídas 15 molas de ar e dispostas 3 em cada estágio do MNP, conforme Figura 3. Uma grande vantagem dessa mola é a possibilidade de controle de sua constante elástica $k$ através do aumento ou da diminuição da pressão interna $P$, independentemente em cada haste (FERREIRA, 2014).

\subsection{Funções de transferência}

O MNP possui cinco graus de liberdade acoplados na direção vertical (z) que, como um oscilador harmônico acoplado, apresenta as relações:

$$
\begin{gathered}
m_{i} \ddot{z}_{i}=-k_{i}\left(z_{i}-z_{i-1}\right)+k_{i+1}\left(z_{i+1}-z_{i}\right) \\
-c_{i}\left(\dot{z}_{i}-\dot{z}_{i-1}\right) \\
+c_{i+1}\left(\dot{z}_{i+1}-\dot{z}_{i}\right)
\end{gathered}
$$


onde $\mathrm{i}=1,2,3,4,5$ representa o estágio do sistema, $\mathrm{m}_{\mathrm{i}}$ são as massas, $\mathrm{k}_{\mathrm{i}}$ são as constantes de mola verticais, $c_{i}=\left(m_{i} k_{i}\right)^{1 / 2} / Q$ são os coeficientes de amortecimento, onde $Q$ é o fator de qualidade mecânico; com $k_{6}, z_{6}$ e $c_{6}$ nulos. No gráfico da Figura 4 pode-se ver a função de transferência $\left|z_{5} / z_{0}\right|$ obtida a partir dessas equações, onde $z_{5}$ é a amplitude de oscilação da massa do último estágio e $z_{0}$ a amplitude de oscilação do solo, isto é, de entrada.

Figura 4 - Função de transferência na direção de oscilação vertical (z) do MNP, para diferentes fatores de qualidade mecânicos $\mathbf{Q}$.

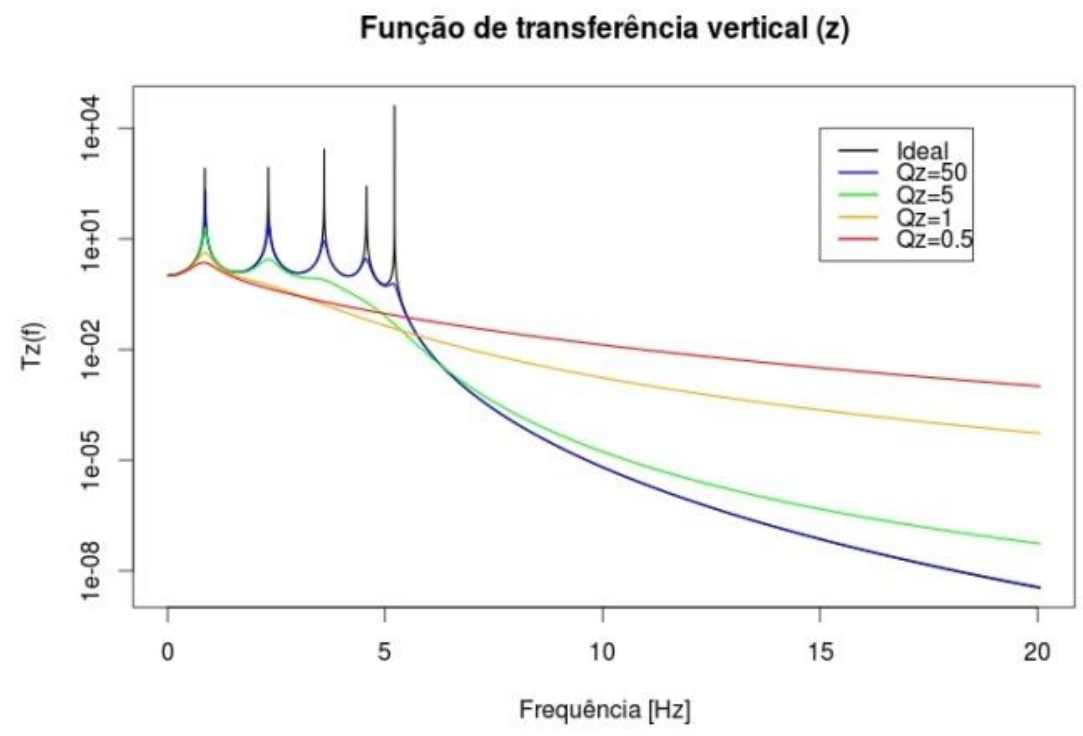

Fonte: Autor.

A solução dessa função foi obtida através do software Maxima ${ }^{5}$, cujo gráfico foi gerado através de um código em linguagem $R^{6}$. Os valores das constantes elásticas $\mathrm{k}_{\mathrm{i}}$ foram estimados a partir das características dinâmicas contidas no manual técnico do fole das molas de ar, dos valores das massas $m_{i}$ e da análise das medidas de vibração de um protótipo. A transferência de oscilação vertical obtida para $Q>50$ é de, aproximadamente, $6,5 \times 10^{-6} \mathrm{em} 10 \mathrm{~Hz}$. Considerando um local silencioso, isto é, $z=10^{-7} \mathrm{~cm} \mathrm{~Hz}^{-1 / 2}$, em $10 \mathrm{~Hz}$ (SAULSON, 1994), a densidade espectral de ruído da posição vertical desse sistema é de $z=6,5 \times 10^{-13} \mathrm{~cm} \mathrm{~Hz}^{-1 / 2}$, o que reafirma $a$ importância do papel de isoladores verticais.

\subsection{Medições}

\footnotetext{
${ }^{5}$ Veja mais detalhes em http://maxima.sourceforge.net/ (acessado em 19/07/2016).

${ }^{6}$ Veja mais detalhes em https://www.r-project.org/ (acessado em 19/04/2016).
} 
Figura 5 - Arranjo experimental para medições de oscilações verticais do MNP, com detalhes mostrando ímã e bobina (quadro da esquerda), sismômetros (quadro da direita) e digitalizadores (em cima da mesa).

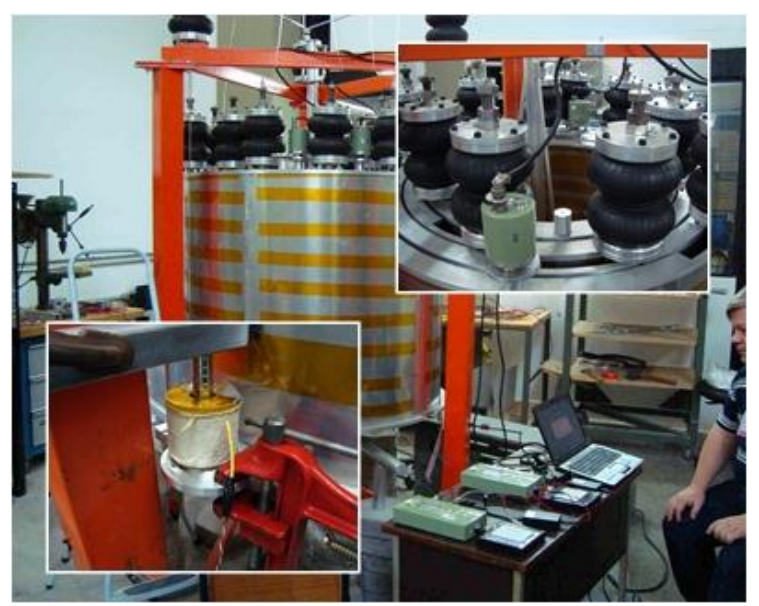

Fonte: Autor.

As medidas e registros das amplitudes de vibração verticais do MNP com as molas de ar foram realizadas utilizando dois sismômetros Trillium Compact ${ }^{7}$, conectados a dois digitalizadores Taurus $^{8}$. Os cilindros mais externos do MNP foram submetidos a forças senoidais verticais através de um sistema de bobinas ligadas a uma fonte $\mathrm{AC}$, que varreu frequências de 1 a $20 \mathrm{~Hz}$, e ímãs de neodímio. É possível ver este arranjo experimental na Figura 5.

A razão entre as amplitudes dos estágios 5 e 1, para o movimento vertical, é apresentada na Figura 6. A diferença entre os dois conjuntos de dados é que os sismômetros foram trocados de posição, isto é, o sismógrafo que estava no estágio 1 foi colocado no estágio 5 e vice-versa.

\subsection{Discussões dos resultados e perspectivas}

As medidas apresentadas na Figura 6 não concordam com as curvas teóricas da Seção 2.3. Esse problema pode estar relacionado a um ou mais dos seguintes fatos: (a) as molas de ar estão longe de serem ideais: apresentam "imperfeições" claras. Foi verificado que elas executam oscilações laterais quando sujeitas a forças impulsivas. Estas inclinações forçam a haste do MNP tocar no tubo interno, causando um curtocircuito mecânico, além de um provável $Q$ mecânico muito baixo; (b) o protótipo do MNP possui hastes muito espessas para o peso total do sistema, que podem apresentar frequências de ressonância menores que $10 \mathrm{~Hz}$; (c) há imperfeições na montagem do sistema que podem causar ou contribuir para o mau desempenho. Uma destas corresponde ao espaço para passagem da haste no orifício do flange do cilindro ser muito estreito, o que também poderia inserir ruído através do atrito da

\footnotetext{
7 Veja mais detalhes em http://www.nanometrics.ca/seismology/products/trillium-compact (acessado em 19/07/2016).

${ }^{8}$ Veja mais detalhes em http://www.nanometrics.ca/seismology/products/taurus (acessado em 19/07/2016)" no termo "Taurus.
} 
haste com este orifício.

Figura 6 - Medidas de oscilações verticais do MNP.

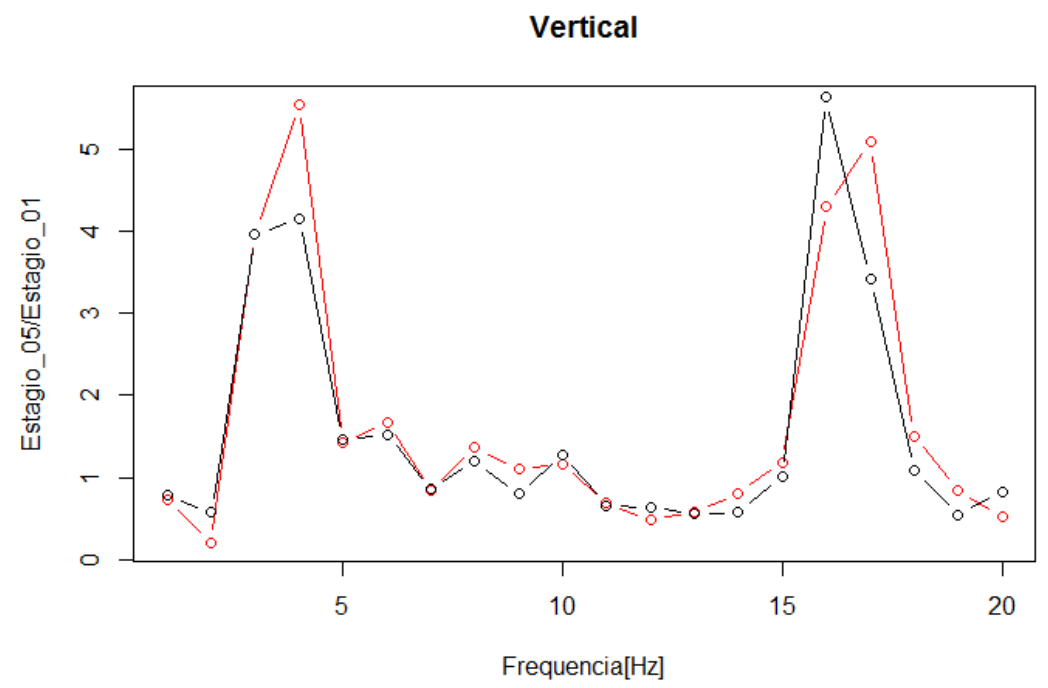

Fonte: Autor

Investigando esses três casos, o GWINPE propôs para cada um deles: (a) novos tipos de molas para o isolamento vertical: denominadas anti-molas geométricas (STOCHINO, 2007), cujo arranjo geométrico de suas lâminas diminui a frequência de ressonância, sem perda de capacidade de carga em relação a uma mola convencional. Feitas de metal, podem ser utilizadas em baixas temperaturas (proposta do LIGO Voyager, para redução do ruído térmico). Um conjunto destas molas já está sendo desenvolvido pelos autores deste trabalho, que substituirá as molas de ar. Os resultados dos novos testes serão apresentados em uma próxima publicação (b) 0 uso de fios de uma liga de titânio-vanádio ou music wire no lugar das hastes se mostra promissor para resolver esse problema. (c) Algumas "imperfeições" visíveis na montagem que já foram corrigidas, tais como a apresentada na Figura 7, cujo buraco para passagem da haste era muito pequeno, permitindo o toque e inserindo-lhe vibrações. 
Figura 7. Detalhe da passagem da haste pelo orifício, cujo contato causava vibrações indesejáveis, e, portanto, foi corrigido.

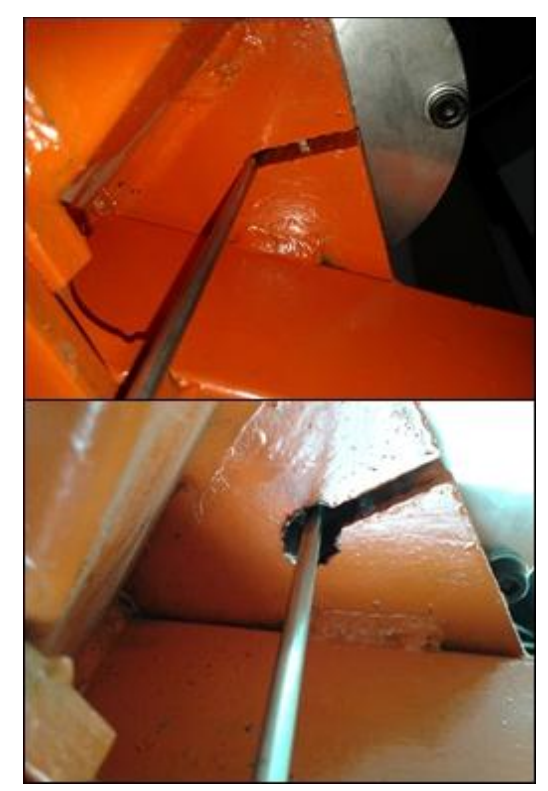

Fonte: Autor

\section{CONCLUSÃO}

Os resultados teóricos do sistema de pêndulos multi-aninhados (MNP) para o isolamento vertical, obtidos por meio das funções de transferência, se mostram promissores para o isolamento vibracional sísmico da futura geração de detectores interferométricos de ondas gravitacionais, como o LIGO Voyager.

Contudo, as medições dos primeiros testes, realizados com molas de ar como isoladores verticais, não são satisfatórias. Elas apresentaram ressonâncias indesejáveis em altas frequências (> $10 \mathrm{~Hz}$ ). A principal perspectiva para melhorar esses resultados é o desenvolvimento de molas especiais, denominadas anti-molas geométricas. Capazes de suportar temperaturas criogênicas e cargas relativamente altas, um efeito da disposição geométrica das lâminas dessa mola, reduzir a frequência de ressonância.

\section{AGRADECIMENTOS}

O GWINPE agradece o Instituto de Geofísica da Universidade de São Paulo e os pesquisadores Marcelo Assumpção, Felipe Neves e Luiz Gallhardo, pelo empréstimo e instruções de uso dos sismômetros e digitalizadores que possibilitaram as medições, além do técnico Marcos Okada, do INPE, que contribuiu com a montagem do aparato 
experimental. O grupo também agradece às agências CAPES e FAPESP (números 12/22752-1 e 14/15370-0), pelo financiamento da pesquisa.

\section{REFERENCIAS}

AASI, J. et al. Advanced ligo. Classical and Quantum Gravity, v. 32, n. 7, 2015.

ABBOTT, B. P. et al. Observation of gravitational waves from a binary black hole merger. Phys. Rev. Lett.,, v. 116, p. 061102-1 - 061102-16, 2016.

CONSTANCIO JÚNIOR, M. Multi-Nested Pendula: Um novo conceito em isolamento vibracional para a terceira e quarta geração do LIGO. São José dos Campos. 2013, 85f. Dissertação (Mestrado em Astrofísica) - Instituto Nacional de Pesquisas Espaciais (INPE), São José dos Campos, 2013.

FERREIRA, E. C. Molas de gás para o sistema de pêndulos multi-aninhados para futuras gerações do LIGO e desenvolvimento dos transdutores da antena Schenberg. São José dos Campos, 2014. 81f. Dissertação (Mestrado em Astrofísica) - Instituto Nacional de Pesquisas Espaciais (INPE), São José dos Campos, 2014.

LIGO-T1400316-V5. Instrument science white paper. (Relatório técnico científico da colaboração), LIGO Scientific Collaboration, 2015.

MAXIMA. Maxima, a Computer Algebra System. 2016. Disponível em: http://maxima.sourceforge.net/>. Acesso em: 19 jul. 2016.

NANOMETRICS. Trillium Compact Broadband Seismometer. 2016. Disponível em: <http://www.nanometrics.ca/seismology/products/trillium-compact>. Acesso em: 19 jul. 2016.

NANOMETRICS. Taurus Digital Seismograph.2016. Disponível em: <http://www.nanometrics.ca/seismology/products/taurus>. Acesso em: 19 jul. 2016.

R-PROJECT. The $\mathbf{R}$ Project for Statistical Computing. 2016. Disponível em: <https://www.r-project.org/>. Acesso em: 19 abr. 2016.

SATHYAPRAKASH, B.; SCHUTZ, B. Physics, astrophysics and cosmology with gravitational waves. Living Reviews in Relativity, v. 12, p. 2, 2009.

SAULSON, P. R. Fundamentals of interferometric gravitational wave detectors. Singapore: World Scientific Publishers, 1994.

STOCHINO, A. The HAS SAS seismic isolation system for the advanced LIGO gravitational wave interferometers. Pisa, 2007. 132f. Tese (Doutorado em Matemática e Física) — Università di Pisa, Pisa, 2007. 